\title{
Mental Health and Fieldwork
}

Calla Hummel, University of Miami

Dana El Kurd, Doha Institute for Graduate Studies

\section{ABSTRACT Researchers discuss the logistics of successful fieldwork but not the mental health considerations that fieldwork and the research process introduce. Successful fieldwork and fruitful academic careers hinge on acknowledging and managing our mental health. We discuss peer-support networks, secondary trauma, coping skills, therapy, and researchers' mental health options before, during, and after fieldwork.}

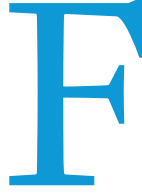

ieldwork-leaving your home institution to acquire data as part of a research project (Kapiszewski, MacLean, and Read 2015, 8)—can bring new stressors because it involves physically leaving daily support structures and entering unfamiliar situations. We argue that political scientists should discuss and include their mental health concerns in their fieldwork preparations. When fieldwork removes researchers from their support structures, we posit that the field-research process can introduce isolation and stress that may exacerbate preexisting mental health conditions (Kapiszewski, MacLean, and Read 2015). When fieldwork involves violent situations or topics, researchers may experience trauma (Loyle and Simoni 2017; Nordstrom and Robben 1995). We use research from anthropology, psychology, and political science to suggest that researchers can manage these stressors by setting up support structures and coping skills before, during, and after fieldwork.

The structures and skills that support mental health during fieldwork also can support it during an academic career (Loyle and Simoni 2017). We suggest that healthy fieldwork practices can spill over to other parts of our professional lives, particularly in situations in which we leave support structures and enter potentially isolating or traumatic environments, such as starting a graduate program or moving for an academic job (Dutt-Ballerastadt 2020; Grollman 2015).

Political science publications cover research design and logistics, such as Kapiszewski, MacLean, and Read's (2015) thorough guide to implementing field research and Gerber and Green's (2012) handbook on field experiments. Kapiszewski, MacLean, and Read (2015, 57-59) included survey questions and discussions about common hardships and researchers' emotional experiences. Moreover, Loyle and Simoni (2017) directly addressed mental health and fieldwork by discussing how fieldwork can expose researchers to secondary trauma and how researchers can address it. Psychologists and anthropologists have incorporated these concerns into research design and training (Nordstrom and

Calla Hummel (iD is assistant professor of political science at the University of Miami. She can be reached at chummel@miami.edu.

Dana El Kurd (D) is assistant professor in the Critical Security Studies Program at the Doha Institute for Graduate Studies. She can be reached at delkurd@dohainstitute.edu.qa
Robben 1995; van der Merwe and Hunt 2019). This article is an extension of the work on research design and implementation to encourage discussions about mental health in graduate training and among fieldworkers.

\section{MENTAL HEALTH AND ACADEMIA}

Academics, universities, and professional associations have discussed "a mental health crisis" (Evans et al. 2018, 282) in academia. Mental health issues disproportionately affect academics who are untenured, in training, and/or a minority in their field (Evans et al. 2018; Levecque et al. 2017; Smith et al. 2007). Evans et al. (2018) surveyed graduate students worldwide and documented mental health issues at rates of more than six times the overall population. About $40 \%$ of graduate students reported anxiety and $40 \%$ reported depression. Evans et al.'s (2018) findings corroborated individual university surveys that found that approximately $50 \%$ of graduate students suffer from anxiety, depression, stress, and other mental health issues (Evans et al. 2018; Levecque et al. 2017). Evans et al. (2018) and Levecque et al. (2017) concluded that academia's professional pressures lead to a high incidence of mental health issues.

Despite more sector-wide conversations about mental health and more institutional resources for treatment (Woolston 2018), few political science venues discuss the mental health challenges of conducting research. Fieldwork warrants its own discussion because most political scientists conduct fieldwork away from the networks of family, friends, and medical providers who support their mental health (Kapiszewski, MacLean, and Read 2015). Some fieldwork topics-including civil war, gendered and racist oppression, and state repression-are particularly likely to expose researchers to violence and incidents of death, putting them at risk for trauma (Loyle and Simoni 2017; Nordstrom and Robben 1995).

Psychologists incorporate mental health, self-care, and traumatology into academic and practitioner training (Knight 2013; Stamm 1995). Anthropologists discuss risks and mental health in ethnographic training (e.g., Nordstrom and Robben 1995), and some sociologists include their struggles and trauma in methodological discussions and appendices (e.g., Contreras 2013). Political science trails behind, even as we encourage and reward risky fieldwork (Driscoll and Schuster 2017). For example, CroninFurman and Lake (2018) noted that weak state capacity and crises 
in conflict zones enable researchers to gain access in ways that would be frowned on (at best) in other contexts. Access to vulnerable populations, including children, can add a new angle to a researcher's project while exploiting subjects (Sukarieh and Tannock 2012). Yet, such behavior often is rewarded because it results in novel data.

When political scientists address mental health and fieldwork, it often is tangential. Sriram et al. (2009), for instance, included a section on surviving research and the impact of fieldwork on researcher behavior, but they did not directly address mental health. When researchers discuss mental health, as in Wood (2006), they focus on how the stress of fieldwork environments inhibits research, with less focus on how the environment impacts them. An exception is Loyle and Simoni's (2017) PS article, which identified research-related trauma and discussed how to manage it. We argue that political science training and institutions need to engage the connections among trauma, fieldwork, and existing mental health concerns.
Another affordable and portable option for fieldworkers are mental health workbooks, which contain exercises for managing conditions such as depression and anxiety. One author uses Mind Over Mood (Greenberger and Padesky 2015), which includes guided journaling, charts for tracking emotions, and exercises such as developing an exposure plan for anxiety.

This author works with a therapist and her support network to prevent relapse into addiction when she is overwhelmed or isolated, which can occur when she moves for work, has a large project to complete alone, or starts fieldwork. Before starting a fieldwork project, she lists activities in advance that she can do to stay healthy and prevent relapse. Her planning is condensed in Safety Card \#2 (table 2).

We recommend that researchers have discussions with their support people about how they can receive support before giving them information in this "cheat-sheet" format. As political scientists, we base these suggestions on our experiences; however, our experience and the options we suggest are not substitutes for

\section{We argue that political science training and institutions need to engage the connections among trauma, fieldwork, and existing mental health concerns.}

\section{MENTAL HEALTH AND FIELDWORK}

Many of us come to fieldwork with chronic mental health conditions (Evans et al. 2018; Smith et al. 2007). Academic pressure can exacerbate these conditions, and fieldwork includes the pressure of implementing a large project with little external structure (Kapiszewski, MacLean, and Read 2015). We use existing research to suggest that research questions, research designs, and social identities influence our mental health during fieldwork.

\section{Managing Existing Mental Health Conditions}

If researchers have existing mental health treatment protocols at their home institution, they have options for continuing medication, therapy, and other treatments at their field site. For example, one of the authors manages chronic anxiety and addiction. She takes medications with her and ensures that she can get them through customs when leaving the United States. She has video appointments with her therapist and sends her family information about where she will be, what she is doing, and emergency contacts. We have condensed the author's planning into Safety Card \#1 (table 1), which helps her think through health logistics before research. It also centralizes information for support people.

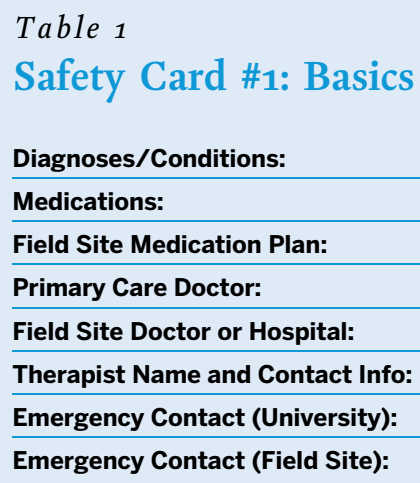

medical assistance. We recommend that researchers seek assistance from other sources and medical professionals.

\section{Therapy}

Being proactive about mental health can protect us from further stress, and one established option is therapy (Hargrave, Scott, and McDowall 2006). Therapists teach evidence-based tools for managing anxiety, depression, and other issues. These professionals can confidentially monitor our mental health throughout our career.

Many therapists offer video sessions for traveling clients, which is helpful when we do fieldwork away from home. Moreover, a growing number of apps offer therapy through a client's smartphone or texting, which can be useful during fieldwork (Firth et al. 2017a). These apps have proven effective in managing depression and anxiety and in developing healthy mental habits (Chandrashekar 2018; Firth et al. 2017b).

\section{Peer-Support Networks}

Studies on fieldwork in authoritarian contexts note the importance of formal support networks in maintaining the safety and

Table 2

Safety Card \#2: Information for Support People

\section{I manage:}

\section{My coping strategies are:}

My symptoms are:

If you are worried about me, ask me:

If I am struggling, it helps if you:

If I am struggling, remind me to:

Please do not:

If you cannot contact me: 
security of researchers, as well as that of their interlocutors (Lake and Parkinson 2017). However, this literature does not emphasize building networks for maintaining mental health, a strategy that can apply to fieldworkers in all contexts. This option is corroborated by research on the benefits of peer-support groups (Cassese and Holman 2018; Trippany, White Kress, and Wilcoxon 2004).

We found that there are two components to our peer-support networks: field-site peers and peers who know our regular routines. Researchers can create a group of friends and contacts, local academics, and other researchers at a field site, whether or not the site is home for the researcher. Researchers can reach out to organizations, research centers, and university departments before fieldwork to affiliate with them, or ask their academic networks for contacts who live and/or conduct research in the area. These networks enrich research and establish academic support networks once fieldwork starts. They are crucial to maintaining the well-being of researchers, particularly in authoritarian or violent environments (Parkinson 2018). experiences. Our peer network, plus our therapists, is how we process secondary trauma from hearing about other people's trauma as part of our research. This mutual-support group helps researchers maintain a connection to their usual support structure, which also helps them understand and process challenges that arise during fieldwork.

\section{Secondary Trauma}

Fieldwork may expose researchers to secondary trauma. People whose jobs expose them to trauma-including researchers-can experience traumatic stress (Browne, Evangeli, and Greenberg 2012; Cieslak et al. 2014). Van der Merwe and Hunt (2019) and Goldenberg (2002) found that field researchers who listen to research participants' stories of trauma may develop secondary traumatic stress-that is, they develop traumatic-stress symptoms after working with people who have experienced violence or death (Cieslak et al. 2014; Stamm 1995). Loyle and Simoni $(2017,141)$ called this "research-related trauma" and extended this work to political scientists.

\section{Our peer network, plus our therapists, is how we process secondary trauma from hearing about other people's trauma as part of our research.}

Researchers can establish a routine with their field peers through coffee dates, telephone calls, writing groups, and events. Vital in this is at least one person whom researchers can ask about informal rules and important logistics, such as doctor recommendations and bureaucratic issues. This group of people is the core of a fieldwork support structure. Their friendship and advice on rules and behavior are important resources and they help us process our experiences (Trippany, White Kress, and Wilcoxon 2004).

For example, one author experienced sexual harassment during field research from the leaders of an organization with whom she was working. She reported the harassment to her adviser and friends at her home institution and her field site. Those at her home institution tried to help but were unsure what to do. Her local peers, however, had useful and concrete advice about addressing sexual harassment, managing it, and when to leave. The advice helped her take better precautions, address some
Witnessing and listening to experiences of injustice can lead to secondary trauma (Goldenberg 2002; van der Merwe and Hunt 2019). Even in a safe environment, many of our questions can elicit difficult answers, and researchers should process those traumatic stories, experiences, and conditions that the people with whom we work share with us (Scheper-Hughes 1992; Trippany, White Kress, and Wilcoxon 2004). For example, when one author interviewed street vendors about their lives and choices, her questions elicited stories of slavery, assault, police brutality, and domestic violence. She subsequently experienced fatigue, anxiety, reduced trust, and fear, and needed to take frequent breaks from research. The other author's research on repression in Palestine involved interviews with Palestinians who had suffered incarceration, physical abuse, and political repression for their views and activities. One interviewee described how his 15-year-old son had been burned to death by Israeli settlers two houses down

\section{Peer-support networks can help establish reasonable goals; recalibrate plans; and address feelings of stress, anxiety, and failure if the researcher falls short of initial fieldwork goals.}

behaviors directly, and redirect other parts of her fieldwork. The other author experienced politically motivated legal issues regarding her entry to the field site. Her fieldwork took place in an environment with rampant repression. Without her local support structure and its ability to safely intervene on her behalf, it would have been impossible to navigate the state's coercive apparatus or the local bureaucracy. The local support structure also helped alleviate the stress and fear arising from fieldwork under a repressive, authoritarian regime and military occupation.

Field researchers can cultivate a group of people who do fieldwork and are familiar with their usual routines, institutions, and support structure. Vital in this is at least one person whom the researcher trusts enough to call or text to process difficult from where the author was staying. Israeli forces physically abused and arrested people that she interviewed during her stay, and she witnessed their families' hardship as they fought charges. These experiences induced secondary trauma that then needed to be treated and resolved through therapy, journaling, and peer-support networks.

Psychologists and others who research trauma find that training, preparing for, and treating that stress as it happens alleviates the impact (Knight 2013; Loyle and Simoni 2017; Stamm 1995). Field researchers report more positive than negative effects of working with trauma, particularly when institutions and researchers take steps to alleviate the negative effects (Goldenberg 2002; van der Merwe and Hunt 2019). 
Research supports actively processing feelings and experiences through journaling (Goldenberg 2002; Trippany, White Kress, and Wilcoxon 2004; Ullrich and Lutgendorf 2002). Journaling is a lowcost option: a few minutes of unstructured writing in any format can have major benefits (Ullrich and Lutgendorf 2002). Additionally, many field-research methods, including ethnography and interviewing, encourage researchers to take notes about their feelings, impressions, and experiences (Nordstrom and Robben 1995). In this way, researchers can incorporate journaling into their existing habits and protocols.

\section{Managing Expectations and Preventing Burnout}

Clear goals and plans prior to fieldwork set manageable expectations; we follow Kapiszewski, MacLean, and Read's (2015) guidelines on planning and implementing fieldwork. This includes a checklist for setting up fieldwork and a spreadsheet to divide data collection into manageable tasks at the field site. Regardless of how organized a researcher may be, fieldwork may not pan out as productively as intended. In our experience, field researchers overestimate how much they can accomplish. Peer-support networks can help establish reasonable goals; recalibrate plans; and address feelings of stress, anxiety, and failure if the researcher falls short of initial fieldwork goals.

Fieldwork takes time and energy, and researchers can supplement work with leisure and rest to prevent burnout (Trippany, White Kress, and Wilcoxon 2004). Cieslak et al. (2014) found a significant and positive association between secondary trauma and burnout across 41 studies, which is particularly strong for people working in the United States and for women. Researchers can plan to take regular and substantial breaks before, during, and after fieldwork.

One option is the revolutionary nineteenth-century idea of taking two days off a week and establishing a regular workday. One author started the practice of taking two days off every week during dissertation fieldwork. She found that she is consistently more productive and creative when she schedules breaks throughout the day and downtime during the week. Another option is to take time off after fieldwork, resources permitting. We both take breaks or vacations-that is, at least 48 hours away from work to do something for fun or relaxation-before diving into analysis after fieldwork. Trippany, White Kress, and Wilcoxon (2004) recommended that institutions provide regular paid vacations for people who work with trauma.

\section{CONCLUSION}

Fieldwork is integral to political science research. Researchers answer pressing questions about politics by collecting original data through field experiments, ethnographic projects, surveys, and interviews with experts, politicians, and voters. Political scientists report that fieldwork is a professionally and personally rewarding experience (Kapiszewski, MacLean, and Read 2015). Although political scientists discuss the logistics of doing fieldwork well (Gerber and Green 2012; Kapiszewski, MacLean, and Read 2015), they rarely address the particular challenges of mental health during its process. Anthropologists and psychologists build mental health plans into research designs, protocols, and academic training, and we suggest that political scientists follow suit.

Adding mental health discussions into fieldwork planning could have a positive impact on the daily work of political scientists more generally. Specifically, political scientists report feeling overwhelmed, isolated, and traumatized by rejection, racism, sexism, classism, ableism, the job market, dissertations, and advisers (Cassese and Holman 2018; Dutt-Ballerstadt 2020; Kapiszewski, MacLean, and Read 2015). These stressors are doubly intense for women and people of color (Niemann 2012). The options discussed in this article could help political scientists process the stress of our daily activities, particularly those that induce trauma and trigger depression, anxiety, and other mental health issues. Planning for mental health wellness, therefore, is essential to healthier fieldwork experiences and may have positive spillover effects on other aspects of our work.

\section{ACKNOWLEDGMENTS}

We thank Kim Guiler, Luciana Molina Barragán, and Sam Serrano for their support and early conversations on this topic, as well as the Political Science Faculty Seminar at the University of Miami and two anonymous reviewers for their invaluable comments. Any errors are our own. -

\section{REFERENCES}

Browne, Tess, Michael Evangeli, and Neil Greenberg. 2012. "Trauma-Related Guilt and Post-Traumatic Stress Among Journalists." Journal of Traumatic Stress 25 (2): 207-10.

Cassese, Erin C., and Mirya R. Holman. 2018. "Writing Groups as Models for Peer Mentorship among Female Faculty in Political Science.” PS: Political Science $\mathcal{E}$ Politics 51 (2): 401-405

Chandrashekar, Pooja. 2018. "Do Mental Health Mobile Apps Work: Evidence and Recommendations for Designing High-Efficacy Mental Health Mobile Apps." mHealth 4 (6)

Cieslak, Roman, Kotaro Shoji, Allison Douglas, Erin Melville, Aleksandra Luszczynska, and Charles C. Benight. 2014. "A Meta-Analysis of the Relationship Between Job Burnout and Secondary Traumatic Stress Among Workers with Indirect Exposure to Trauma." Psychological Services 11 (1): 75.

Contreras, Randol. 2013. The Stickup Kids: Race, Drugs, Violence, and the American Dream. Berkeley: University of California Press.

Cronin-Furman, Kate, and Milli Lake. 2018. "Ethics Abroad: Fieldwork in Fragile and Violent Contexts." PS: Political Science \& Politics 51 (3): 607-14.

Driscoll, Jesse, and Caroline Schuster. 2017. “Spies Like Us.” Ethnography 19 (3): 411-30.

Dutt-Ballerstadt, Reshmi. 2020. "Institutional Betrayals: In Our Own Words.” Inside Higher Ed, March 6. Available at www.insidehighered.com/advice/2020/03/06/ underrepresented-faculty-members-share-real-reasons-they-have-left-various.

Evans, Teresa M., Lindsay Bira, Jazmin Beltran Gastelum, L. Todd Weiss, and Nathan L. Vanderford. 2018. "Evidence for a Mental Health Crisis in Graduate Education." Nature Biotechnology 36 (3): 282-84

Firth, Joseph, John Torous, Jennifer Nicholas, Rebekah Carney, Simon Rosenbaum, and Jerome Sarris. 2017a. "Can Smartphone Mental Health Interventions Reduce Symptoms of Anxiety? A Meta-Analysis of Randomized Controlled Trials." Journal of Affective Disorders 218:15-22.

Firth, Joseph, John Torous, Jennifer Nicholas, Rebekah Carney, Abhishek Pratap, Simon Rosenbaum, and Jerome Sarris. 2017b. "The Efficacy of Smartphone-Based Mental Health Interventions for Depressive Symptoms: A Meta-Analysis of Randomized Controlled Trials." World Psychiatry 16 (3): 287-98.

Gerber, Alan S., and Donald P. Green. 2012. Field Experiments: Design, Analysis, and Interpretation. New York: W. W Norton \& Company.

Goldenberg, Jennifer. 2002. "The Impact on the Interviewer of Holocaust Survivor Narratives: Vicarious Traumatization or Transformation?" Traumatology 8 (4): 215-31.

Greenberger, Dennis, and Christine A. Padesky. 2015. Mind over Mood: Change How You Feel by Changing the Way You Think. New York: Guilford Publications, Inc.

Grollman, Eric Anthony. 2015. “Trauma on Display.” Inside Higher Ed, September 29 Available at www.insidehighered.com/advice/2015/09/29/essay-traumaassociated-graduate-school.

Hargrave, Petrina A., Kate M. Scott, and John McDowall. 2006. "To Resolve or Not to Resolve: Past Trauma and Secondary Traumatic Stress in Volunteer Crisis Workers." Journal of Trauma Practice 5 (2): 37-55. 
Kapiszewski, Diana, Lauren M. MacLean, and Benjamin L. Read. 2015. Field Research in Political Science: Practices and Principles. Cambridge: Cambridge University Press.

Knight, Carolyn. 2013. "Indirect Trauma: Implications for Self-Care, Supervision, the Organization, and the Academic Institution." The Clinical Supervisor 32 (2): 224-43

Lake, Milli, and Sarah E. Parkinson. 2017. "The Ethics of Fieldwork Preparedness." Political Violence at a Glance, June 5. Available at www.politicalviolenceataglance.org/ 2017/06/05/the-ethics-of-fieldwork-preparedness.

Levecque, Katia, Frederik Anseel, Alain DeBeuckelaer, Johan Van der Heyden, and Lydia Gisle. 2017. "Work Organization and Mental Health Problems in PhD Students." Research Policy 46 (4): 868-79.

Loyle, Cyanne E., and Alicia Simoni. 2017. "Researching under Fire: Political Science and Researcher Trauma." PS: Political Science \& Politics 50 (1): 141-45.

Niemann, Yolanda Flores. 2012. "Lessons from the Experiences of Women of Color Working in Academia." In Presumed Incompetent: The Intersections of Race and Class for Women in Academia, ed. Angela P. Harris, Gabriella Gutiérrezy Muhs, Yolanda Flores Niemann, and Carmen G. Gonzalez, 446-99. Logan: Utah State University Press.

Nordstrom, Carolyn, and Antonius C. G. M. Robben (eds.). 1995. Fieldwork under Fire: Contemporary Studies of Violence and Culture. Berkeley: University of California Press.

Parkinson, Sarah. 2018. "Seeing Beyond the Spectacle: Research on and Adjacent to Violence." In Political Science Research in the Middle East and North Africa: Methodological and Ethical Challenges, ed. Janine A. Clark and Francesco Cavatorta, 73-82. New York: Oxford University Press.

Scheper-Hughes, Nancy. 1992. Death without Weeping: The Violence of Everyday Life in Brazil. Berkeley: University of California Press.
Smith, Carol Klose, D. Fred Peterson, Brian F. Degenhardt, and Jane C. Johnson. 2007 "Depression, Anxiety, and Perceived Hassles Among Entering Medical Students." Psychology, Health \& Medicine 12 (1): 31-39.

Sriram, Chandra, John King, Julie Mertus, Olga Martin-Ortega, and Johanna Herman (eds.). 2009. Surviving Field Research: Working in Violent and Difficult Situations. Philadelphia: Routledge.

Stamm, Beth. 1995. Secondary Traumatic Stress: Self-Care Issues for Clinicians, Researchers, and Educators. Derwood, MD: The Sidran Press.

Sukarieh, Mayssoun, and Stuart Tannock. 2012. "On the Problem of Over-Researched Communities: The Case of the Shatila Palestinian Refugee Camp in Lebanon." Sociology 47 (3): 494-508.

Trippany, Robyn L., Victoria E. White Kress, and S. Allen Wilcoxon. 2004 "Preventing Vicarious Trauma: What Counselors Should Know When Working with Trauma Survivors." Journal of Counseling \& Development 82 (1): 31-37.

Ullrich, Philip M., and Susan K. Lutgendorf. 2002. "Journaling about Stressful Events: Effects of Cognitive Processing and Emotional Expression." Annals of Behavioral Medicine 24 (3): 244-50.

van der Merwe, Amelia, and Xanthe Hunt. 2019. "Secondary Trauma among Trauma Researchers: Lessons from the Field.” Psychological Trauma: Theory, Research, Practice, and Policy 11 (1): 10.

Wood, Elizabeth. 2006. “The Ethical Challenges of Field Research in Conflict Zones.” Qualitative Sociology. 29:373-86.

Woolston, Chris. 2018. "Why Mental Health Matters." Nature Careers 557:129-31. 